\title{
Globally Exponential Stability of Impulsive Neural Networks with Given Convergence Rate
}

\author{
Chengyan Liu, Xiaodi Li, and Xilin Fu \\ Department of Mathematics, Shandong Normal University, Jinan 250014, China \\ Correspondence should be addressed to Xiaodi Li; sodymath@163.com
}

Received 29 November 2012; Accepted 12 April 2013

Academic Editor: Manwai Mak

Copyright (c) 2013 Chengyan Liu et al. This is an open access article distributed under the Creative Commons Attribution License, which permits unrestricted use, distribution, and reproduction in any medium, provided the original work is properly cited.

\begin{abstract}
This paper deals with the stability problem for a class of impulsive neural networks. Some sufficient conditions which can guarantee the globally exponential stability of the addressed models with given convergence rate are derived by using Lyapunov function and impulsive analysis techniques. Finally, an example is given to show the effectiveness of the obtained results.
\end{abstract}

\section{Introduction}

Recently, special interest has been devoted to the dynamics analysis of neural networks due to their potential applications in different areas of science. Particularly, there has been a significant development in the theory of neural networks with impulsive effects [1-9], since such neural networks with impulsive effect can be used as an appropriate description of the phenomena of abrupt qualitative dynamical changes of essential continuous time systems. Based on the theory of impulsive differential equations [10-17], some sufficient conditions guaranteeing the exponential stability are derived [1824]. For example, in [8], the author has obtained a criterion of exponential stability for a Hopfield neural network with periodic coefficients; in [18], by constructing the extended impulsive delayed Halanay inequality and Lyapunov functional methods, authors have got some sufficient conditions ensuring exponential stability of the unique equilibrium point of impulsive Hopfield neural networks with time delays. They all have obtained exponential stability for some kinds of neural networks through different methods. However, most of the existing results about the exponential stability of impulsive neural networks have a common feature that the exponential convergence rate cannot be derived, or derived but not the given one $[8,18,23,24]$. The purpose of this paper is to establish some criteria which can guarantee the globally exponential stability of impulsive neural networks with the given convergence rate by using Lyapunov function and impulsive analysis techniques. This work is organized as follows. In Section 2, we introduce some basic definitions and notations. In Section 3, the main results are presented. In Section 4 , an example is discussed to illustrate the results.

\section{Preliminaries}

Let $\mathbb{R}$ denote the set of real numbers, $\mathbb{R}_{+}$denote the set of nonnegative real numbers, $\mathbb{Z}_{+}$denote the set of positive integers and $\mathbb{R}^{n}$ denote the $n$-dimensional real space equipped with the Euclidean norm $\|\cdot\|$.

Consider the following impulsive neural networks:

$$
\begin{aligned}
\dot{x}_{i}(t)= & -a_{i}(t) x_{i}(t) \\
& +\sum_{j=1}^{n} b_{i j}(t) f_{j}\left(x_{j}(t)\right)+I_{i}(t), \quad t \geq t_{0}, t \neq t_{k}, \\
\left.\Delta x_{i}\right|_{t=t_{k}}= & x_{i}\left(t_{k}\right)-x_{i}\left(t_{k}^{-}\right), \quad k \in \mathbb{Z}_{+}, i \in \Lambda,
\end{aligned}
$$

where $\Lambda=\{1,2, \ldots, n\} . n \geq 2$ corresponds to the number of units in a neural network; the impulse times $t_{k}$ satisfy $0 \leq t_{0}<$ $t_{1}<\cdots<t_{k}<\cdots, \lim _{k \rightarrow \infty} t_{k}=\infty$; $x_{i}$ corresponds to the state of the neurons, $f_{j}$ denotes the measures of response to its incoming potentials of the unit $j$ at time $t ; I_{i}(t)$ is the input of the unit $i$ at time $t . P C[I, \mathbb{R}] \triangleq\left\{\varphi: I \rightarrow \mathbb{R} \mid \varphi\left(t^{+}\right)=\varphi(t)\right.$ for $t \in I, \varphi\left(t^{-}\right)$exists for $t \in I, \varphi\left(t^{-}\right)=\varphi(t)$ for all but points $t_{k} \in$ $I\}$, where $I \subset \mathbb{R}$ is an interval, $\varphi\left(t^{+}\right)$and $\varphi\left(t^{-}\right)$denote the left 
limit and right limit of function $\varphi(t)$, respectively. $a_{i}(t)>0$, $b_{i j}(t), I_{i}(t) \in P C\left[\left[t_{0},+\infty\right), \mathbb{R}\right]$. For given $t_{0}, x\left(t_{0}\right)=x_{0}=\left(x_{1}^{0}\right.$, $\left.x_{2}^{0}, \ldots, x_{n}^{0}\right) \in \mathbb{R}^{n}$, we denote by $x(t)$ the solution of system (1) with initial value $\left(t_{0}, x\left(t_{0}\right)\right)$.

In this paper, we assume that some conditions are satisfied so that the equilibrium point of system (1) does exist, see $[16,17]$. Assume that $x^{\star}=\left(x_{1}^{\star}, x_{2}^{\star}, \ldots, x_{n}^{\star}\right)^{T}$ is an equilibrium point of system (1). Impulsive operator is viewed as perturbation of the point $x^{\star}$ of such system without impulsive effects. We assume that the following impulsive condition holds.

$\left.\left(\mathrm{H}_{0}\right) \Delta x_{i}\right|_{t=t_{k}}=x_{i}\left(t_{k}\right)-x_{i}\left(t_{k}^{-}\right)=\sigma_{i k}\left(x_{i}\left(t_{k}^{-}\right)-x_{i}^{\star}\right), \sigma_{i k} \in$ $\mathbb{R}, i \in \Lambda, k \in \mathbb{Z}_{+}$.

Furthermore, we will assume that the response function $f_{i}$ satisfies the following condition.

$\left(\mathrm{H}_{1}\right) f_{i}$ is globally Lipschizian with Lipschitz constant $l_{i}>0$, that is, $\left|f_{i}\left(s_{1}\right)-f_{i}\left(s_{2}\right)\right| \leq l_{i}\left|s_{1}-s_{2}\right|$, for all $s_{1}, s_{2} \in$ $\mathbb{R}, i \in \Lambda$.

Note that $x^{\star}$ is an equilibrium point of system (1), one can derive from system (1) that the transformation $z_{i}=x_{i}-x_{i}^{\star}$, $i \in \Lambda$ transforms such system into the following system:

$$
\begin{aligned}
\dot{z}_{i}(t)= & -a_{\mathrm{i}}(t) z_{i}(t) \\
& +\sum_{j=1}^{n} b_{i j}(t) F_{j}\left(z_{j}(t)\right), \quad t \geq t_{0}, \quad t \neq t_{k}, \\
z_{i}\left(t_{k}\right)= & \left(1+\sigma_{i k}\right) z_{i}\left(t_{k}^{-}\right), \quad i \in \Lambda, k \in \mathbb{Z}_{+},
\end{aligned}
$$

where $F_{j}\left(z_{j}(t)\right)=f_{j}\left(x_{j}^{\star}+z_{j}(t)\right)-f_{j}\left(x_{j}^{\star}\right)$, and from the condition $\left(\mathrm{H}_{1}\right)$, it holds that $\left\|F_{j}\left(z_{j}(t)\right)\right\| \leq l_{j}\left\|z_{j}(t)\right\|, j \in \Lambda$.

Furthermore, let $y_{i}(t)=z_{i}(t) e^{\alpha\left(t-t_{0}\right)}, i \in \Lambda$, then system (2) becomes as follows:

$$
\begin{aligned}
\dot{y}_{i}(t)= & \left(\alpha-a_{i}(t)\right) y_{i}(t) \\
& +e^{\alpha\left(t-t_{0}\right)} \sum_{j=1}^{n} b_{i j}(t) F_{j}\left(y_{j}(t) e^{-\alpha\left(t-t_{0}\right)}\right), \quad t \geq t_{0}, \quad t \neq t_{k}, \\
y_{i}\left(t_{k}\right)= & \left(1+\sigma_{i k}\right) y_{i}\left(t_{k}^{-}\right), \quad i \in \Lambda, k \in \mathbb{Z}_{+} .
\end{aligned}
$$

To prove the stability of $x^{\star}$ of system (1), it is equal to prove the stability of the zero solution of system (2), and also equal to the boundedness of system (3).

In the following, the notion $A^{T}$ means the transpose of a square matrix $A$. We will use the notation $A>0$ (or $A<0$, $A \geq 0, A \leq 0)$ to denote that the matrix $A$ is a positive definite (negtive definite, positive semidefinite, an negative semidefinite) marix.

Let $y(t)=\left(y_{1}(t), y_{2}(t), \ldots, y_{n}(t)\right)^{T}, A(t)=\operatorname{diag}\left[a_{1}(t)\right.$, $\left.a_{2}(t), \ldots, a_{n}(t)\right], B(t)=\left(b_{i j}(t)\right)_{n \times n}, L=\operatorname{diag}\left[l_{1}, l_{2}, \ldots, l_{n}\right]$, $I=\operatorname{diag}[1,1, \ldots, 1], D_{k}=\operatorname{diag}\left[1+\sigma_{1 k}, 1+\sigma_{2 k}, \ldots, 1+\sigma_{n k}\right]$, $F(y)=\left(F_{1}\left(y_{1}\right), F_{2}\left(y_{2}\right), \ldots \text {, and } F_{n}\left(y_{n}\right)\right)^{T}$ then system (3) with initial condition becomes as follows:

$$
\begin{aligned}
\dot{y}(t)= & (\alpha I-A(t)) y(t) \\
& +e^{-\alpha\left(t-t_{0}\right)} B(t) F\left(y(t) e^{-\alpha\left(t-t_{0}\right)}\right), \quad t \geq t_{0}, t \neq t_{k}, \\
y\left(t_{k}\right)= & D_{k} y\left(t_{k}^{-}\right), \quad i \in \Lambda, k \in \mathbb{Z}_{+} .
\end{aligned}
$$

We introduce a definition as follows.
Definition 1. Assume $x^{\star}=\left(x_{1}^{\star}, x_{2}^{\star}, \ldots, x_{n}^{\star}\right)^{T} \in \mathbb{R}^{n}$ is the equilibrium point of system (1), then the equilibrium point $x^{\star}$ of system (1) is said to be globally exponential stable with given convergence rate $\alpha>0$. If for any initial data $x\left(t_{0}\right)=x_{0} \in \mathbb{R}$, there exists a constant $M \geqslant 1$, such that

$$
\left\|x\left(t, t_{0}, x_{0}\right)-x^{\star}\right\| \leqslant\left\|x_{0}-x^{\star}\right\| M e^{-\alpha\left(t-t_{0}\right)}, \quad t \geqslant t_{0} .
$$

From the transformation $z_{i}=x_{i}-x_{i}^{\star}, i \in \Lambda$, and $z\left(t_{0}\right)=$ $z_{0}=x_{0}-x^{\star}$, the globally exponential stability of the equilibrium point $x^{\star}$ of system (1) can be transformed into the globally exponential stability of trivial solution of system (2), so (5) can be rewritten as follows:

$$
\left\|z\left(t, t_{0}, z_{0}\right)\right\| \leq\left\|z\left(t_{0}\right)\right\| M e^{-\alpha\left(t-t_{0}\right)}, \quad t \geqslant t_{0} .
$$

Furthermore, form the transformation $y(t)=z\left(t, t_{0}\right.$, $\left.z_{0}\right) e^{\alpha\left(t-t_{0}\right)}, i \in \Lambda$, the globally exponential stability of trivial solution of system (2) can be transformed into the boundedness of the solution of system (4) and it can be rewritten as follows:

$$
\|y(t)\| \leq\left\|y\left(t_{0}\right)\right\| M, \quad t \geqslant t_{0} .
$$

\section{Main Results}

Theorem 2. Given constant $\alpha>0$. The equilibrium point of the system (1) is globally exponentially stable with the given convergence rate $\alpha$, if the conditions $\left(H_{0}\right)$ and $\left(H_{1}\right)$ are fulfilled; moreover, suppose that

(i) $\alpha I-A(t)+B(t) L \leq 0$, for all $t>t_{0}$,

(ii) $\prod_{k=1}^{\infty} \max _{i \in \Lambda}\left(1+\sigma_{i k}\right)<\infty$, and $\sigma_{i k} \geq 0, i \in \Lambda, k \in \mathbb{Z}_{+}$.

Proof. We only need to prove $y(t)=z(t) e^{\alpha\left(t-t_{0}\right)}$ is bounded when $t \geq t_{0}$, where $z(t)=z\left(t, t_{0}, z_{0}\right)$ is a solution of (3) through $\left(t_{0}, z_{0}\right)$.

Consider the Lyapunov function as follows:

$$
V(t)=y(t)^{T} y(t)=\sum_{i=1}^{n} y_{i}^{2}(t) .
$$

Particularly, $V\left(t_{0}\right)=\sum_{i=1}^{n} y_{i}^{2}\left(t_{0}\right)$.

Then from conditions $\left(\mathrm{H}_{0}\right)-\left(\mathrm{H}_{1}\right)$ and $(\mathrm{i})$, we get the upper right-hand derivative of $V(t)$ along the solutions of system (3), for $t \in\left[t_{k}, t_{k+1}\right), k \in \mathbb{Z}_{+}$

$$
\begin{aligned}
D^{+} V(t) & =2 \sum_{i=1}^{n} y_{i}(t) \dot{y}_{i}(t) \\
& =2 \sum_{i=1}^{n} z_{i}(t) e^{2 \alpha\left(t-t_{0}\right)}\left[\dot{z}_{i}(t)+\alpha z_{i}(t)\right] \\
& =2 \sum_{i=1}^{n} z_{i}(t) e^{2 \alpha\left(t-t_{0}\right)} \\
& \times\left[-a_{i}(t) z_{i}(t)+\sum_{j=1}^{n} b_{i j}(t) F_{j}\left(z_{j}(t)\right)+\alpha z_{i}(t)\right]
\end{aligned}
$$




$$
\begin{aligned}
& =2 \sum_{i=1}^{n} e^{2 \alpha\left(t-t_{0}\right)} \\
& \quad \times\left[\left(\alpha-a_{i}(t)\right) z_{i}^{2}(t)+z_{i}(t) \sum_{j=1}^{n} b_{i j}(t) F_{j}\left(z_{j}(t)\right)\right] \\
& \leq 2 \sum_{i=1}^{n} e^{2 \alpha\left(t-t_{0}\right)} \\
& \quad \times\left[\left(\alpha-a_{i}(t)\right) z_{i}(t)^{2}+z_{i}(t) \sum_{j=1}^{n} b_{i j}(t) l_{j}\left\|z_{j}(t)\right\|\right] \\
& =2 \sum_{i=1}^{n} e^{2 \alpha\left(t-t_{0}\right)} z_{i}^{2}(t)\left(\alpha-a_{i}(t)\right) \\
& \quad+2 \sum_{i=1}^{n} e^{2 \alpha\left(t-t_{0}\right)} z_{i}(t) \sum_{j=1}^{n} b_{i j}(t) l_{j}\left\|z_{j}(t)\right\| \\
& =2 y(t)^{T}[\alpha I-A(t)+B(t) L] y(t) \\
& \leq 0,
\end{aligned}
$$

which implies the functional $V(t)$ is nonincreasing for $t \in$ $\left[t_{k} t_{k+1}\right), k \in \mathbb{Z}_{+}$. By condition (ii), it holds that

$$
\begin{aligned}
V\left(t_{k}\right)=\sum_{i=1}^{n} y_{i}^{2}\left(t_{k}\right) & =\sum_{i=1}^{n} z_{i}^{2}\left(t_{k}\right) e^{2 \alpha\left(t_{k}-t_{0}\right)} \\
& =\sum_{i=1}^{n} e^{2 \alpha\left(t-t_{0}\right)}\left[z_{i}\left(t_{k}^{-}\right)+J_{i}\left(z_{i}\left(t_{k}^{-}\right)\right)\right]^{2} \\
& =\sum_{i=1}^{n} e^{2 \alpha\left(t-t_{0}\right)}\left(1+\sigma_{i k}\right)^{2} z_{i}^{2}\left(t_{k}^{-}\right) \\
& \leq \max _{i \in \Lambda}\left(1+\sigma_{i k}\right)^{2} V\left(t_{k}^{-}\right) .
\end{aligned}
$$

For any $t \in\left[t_{0}, t_{1}\right)$, since $V(t)$ is nonincreasing, it holds that $V(t) \leq V\left(t_{0}\right)$; moreover,

$$
V\left(t_{1}\right) \leq \max _{i \in \Lambda}\left(1+\sigma_{i 1}\right)^{2} V\left(t_{1}^{-}\right) \leq \max _{i \in \Lambda}\left(1+\sigma_{i 1}\right)^{2} V\left(t_{0}\right) .
$$

Similarly, for any $t \in\left[t_{1}, t_{2}\right)$, it holds that $V(t) \leq V\left(t_{1}\right) \leq$ $\max _{i \in \Lambda}\left(1+\sigma_{i 1}\right)^{2} V\left(t_{0}\right)$, and

$$
\begin{aligned}
V\left(t_{2}\right) & \leq \max _{i \in \Lambda}\left(1+\sigma_{i 2}\right)^{2} V\left(t_{2}^{-}\right) \\
& \leq \max _{i \in \Lambda}\left(1+\sigma_{i 2}\right)^{2} \max _{i \in \Lambda}\left(1+\sigma_{i 1}\right)^{2} V\left(t_{0}\right) .
\end{aligned}
$$

Thus, it can be deduced that for $t \in\left[t_{k}, t_{k+1}\right), k \in \mathbb{Z}_{+}$,

$$
V(t) \leq \prod_{j=1}^{k} \max _{i \in \Lambda}\left(1+\sigma_{i j}\right)^{2} V\left(t_{0}\right) .
$$

Hence, we obtain that for any $t \geq t_{0}$,

$$
V(t) \leq \prod_{j=1}^{\infty} \max _{i \in \Lambda}\left(1+\sigma_{i j}\right)^{2} V\left(t_{0}\right),
$$

which implies that

$$
\|y(t)\| \leq M\left\|y\left(t_{0}\right)\right\|, \quad t \geq t_{0},
$$

where $M=\prod_{k=1}^{\infty} \max _{i \in \Lambda}\left(1+\sigma_{i k}\right)<\infty$.

The proof of Theorem 2 is complete.

Remark 3. Most of the existing results about the exponential stability of impulsive neural networks cannot effectively control the convergence rate. It is interesting to see that Theorem 2 can guarantee the globally exponential stability of impulsive neural networks with the given convergence rate.

Remark 4. In particular, if $A(t) \equiv A, B(t) \equiv B$ in Theorem 2, where $A, B$ are constant matrices, then condition $\alpha I-A+B L<$ 0 can be easily checked via Matlab.

Theorem 5. Given constant $\alpha>0$. The equilibrium point of the system (1) is globally exponentially stable with the given convergence rate $\alpha$, if the conditions $\left(H_{0}\right)-\left(H_{1}\right)$ are fulfilled; moreover, suppose that

(i) there exists a constant $\lambda>0$, such that $(\alpha+\lambda) I-A(t)+$ $B(t) L<0$, for all $t>t_{0}$,

(ii) $\tau \triangleq \min _{k \in \mathbb{Z}_{+}}\left\{t_{k+1}-t_{k}\right\}, \max _{i \in \Lambda}\left(1+\sigma_{i k}\right) \leq M_{k} e^{\lambda \tau}$, where $1 \leq M_{k}<\infty$ and $\prod_{k=1}^{\infty} M_{k}<\infty, i \in \Lambda, k \in \mathbb{Z}_{+}$.

Proof. We only need to prove that $y(t)=z(t) e^{\alpha\left(t-t_{0}\right)}$ is bounded when $t \geq t_{0}$, where $z(t)=z\left(t, t_{0}, z_{0}\right)$ is a solution of (3) through $\left(t_{0}, z_{0}\right)$.

Consider the Lyapunov function as follows:

$$
V(t)=y(t)^{T} y(t)=\sum_{i=1}^{n} y_{i}^{2}(t) .
$$

In particular, $V\left(t_{0}\right)=\sum_{i=1}^{n} y_{i}^{2}\left(t_{0}\right)$.

Then from conditions $\left(\mathrm{H}_{0}\right)-\left(\mathrm{H}_{1}\right)$ and $(\mathrm{i})$, we get the upper right-hand derivative of $V(t)$ along the solutions of system (3), for $t \in\left[t_{k}, t_{k+1}\right), k \in \mathbb{Z}_{+}$

$$
\begin{aligned}
D^{+} V(t) & =2 \sum_{i=1}^{n} y_{i}(t) \dot{y}_{i}(t) \\
& \leq 2 y(t)^{T}(\alpha I-A(t)+B(t) L) y(t)<-2 \lambda V(t) .
\end{aligned}
$$

Thus,

$$
\begin{array}{r}
V(t)<V\left(t_{k}\right) e^{-2 \lambda\left(t-t_{k}\right)}<V\left(t_{k}\right) e^{-2 \lambda \tau}, \quad t \in\left[t_{k}, t_{k+1}\right), \\
k \in \mathbb{Z}_{+} .
\end{array}
$$


By condition (ii), it holds that

$$
\begin{aligned}
V\left(t_{k}\right)=\sum_{i=1}^{n} y_{i}^{2}\left(t_{k}\right) & =\sum_{i=1}^{n} e^{2 \alpha\left(t-t_{0}\right)}\left(1+\sigma_{i k}\right)^{2} z_{i}^{2}\left(t_{k}^{-}\right) \\
& \leq M_{k}^{2} e^{2 \lambda \tau} \sum_{i=1}^{n} e^{2 \alpha\left(t-t_{0}\right)} z_{i}^{2}\left(t_{k}^{-}\right) \\
& \leq M_{k}^{2} e^{2 \lambda \tau} V\left(t_{k}^{-}\right) .
\end{aligned}
$$

For any $t \in\left[t_{0}, t_{1}\right)$, it holds that $V(t) \leq V\left(t_{0}\right) e^{-2 \lambda \tau}$, moreover

$$
V\left(t_{1}\right) \leq M_{1}^{2} e^{2 \lambda \tau} V\left(t_{1}^{-}\right) \leq M_{1}^{2} V\left(t_{0}\right) \text {. }
$$

Similarly, for any $t \in\left[t_{1}, t_{2}\right)$, it holds that $V(t) \leq V\left(t_{1}\right) e^{-2 \lambda \tau} \leq$ $M_{1}^{2} V\left(t_{0}\right) e^{-2 \lambda \tau}$, and

$$
V\left(t_{2}\right) \leq M_{2} e^{2 \lambda \tau} V\left(t_{2}^{-}\right) \leq M_{2}^{2} M_{1}^{2} V\left(t_{0}\right) .
$$

Without loss of generality, when $t \in\left[t_{k}, t_{k+1}\right), k \in \mathbb{Z}_{+}$, it can be deduced that

$$
V(t) \leq \prod_{j=1}^{k} M_{j}^{2} e^{-2 \lambda \tau} V\left(t_{0}\right) .
$$

Hence, we obtain that for any $t \geq t_{0}$,

$$
V(t) \leq \prod_{j=1}^{\infty} M_{j}^{2} V\left(t_{0}\right),
$$

which implies that

$$
\|y(t)\| \leq M\left\|y\left(t_{0}\right)\right\|, \quad t \geq t_{0},
$$

where $M=\left(\prod_{j=1}^{\infty} M_{j}\right)<\infty$.

The proof of Theorem 5 is complete.

Remark 6. Although Theorem 5 enhances the restriction on condition (i), the impulsive restriction in (ii) is weaker; that is, $\sigma_{i k}$ is not necessary to converge to 0 as $k$ is large enough, provided that the impulsive intervals are not too small.

Theorem 7. Given constant $\alpha>0$. The equilibrium point of the system (1) is globally exponentially stable with the given exponential convergence rate $\alpha$, if the conditions $\left(H_{0}\right)-\left(H_{1}\right)$ are fulfilled; moreover, suppose that

(i) there exists a constant $\lambda>0$, such that $(\alpha-\lambda) I-A(t)+$ $B(t) L<0$, for all $t>t_{0}$,

(ii) $\tau \triangleq \max _{k \in \mathbb{Z}_{+}}\left\{t_{k+1}-t_{k}\right\}$ and $\beta_{k} e^{\lambda \tau} \leq 1$, where $\beta_{k}=$ $\max _{i \in \Lambda}\left(1+\sigma_{i k}\right),-1 \leq \sigma_{i k} \leq 0, i \in \Lambda, k \in \mathbb{Z}_{+}$.

Proof. We only need to prove that $y(t)=z(t) e^{\alpha\left(t-t_{0}\right)}$ is bounded when $t \geq t_{0}$, where $z(t)=z\left(t, t_{0}, z_{0}\right)$ is a solution of (3) through $\left(t_{0}, z_{0}\right)$.

Consider the Lyapunov functional as follows:

$$
V(t)=y(t)^{T} y(t)=\sum_{i=1}^{n} y_{i}^{2}(t) .
$$

Particularly, $V\left(t_{0}\right)=\sum_{i=1}^{n} y_{i}^{2}\left(t_{0}\right)$.
Then from conditions $\left(\mathrm{H}_{0}\right)-\left(\mathrm{H}_{1}\right)$ and $(\mathrm{i})$, we get the upper right-hand derivative of $V(t)$ along the solutions of system (1), for $t \in\left[t_{k}, t_{k+1}\right), k \in \mathbb{Z}_{+}$

$$
\begin{aligned}
D^{+} V(t) & =2 \sum_{i=1}^{n} y_{i}(t) \dot{y}_{i}(t) \\
& =2 y(t)^{T}(\alpha I-A(t)+B(t) L) y(t)<2 \lambda V(t) .
\end{aligned}
$$

Thus,

$$
V(t)<V\left(t_{k}\right) e^{2 \lambda\left(t-t_{k}\right)}<V\left(t_{k}\right) e^{2 \lambda \tau}, \quad t \in\left[t_{k}, t_{k+1}\right), k \in \mathbb{Z}_{+} .
$$

By condition (ii), it holds that

$$
\begin{aligned}
V\left(\mathrm{t}_{k}\right) & =\sum_{i=1}^{n} y_{i}^{2}\left(t_{k}\right) \leq \max _{i \in \Lambda}\left(1+\sigma_{i k}\right)^{2} V\left(t_{k}^{-}\right) \\
& =\beta_{k}^{2} V\left(t_{k}^{-}\right), \quad k \in \mathbb{Z}_{+} .
\end{aligned}
$$

By simple induction, we can prove that for any $t \in\left[t_{k}, t_{k+1}\right)$, $k \in \mathbb{Z}_{+}$,

$$
V(t) \leq \prod_{j=1}^{k} \beta_{j}^{2} e^{2(k+1) \lambda \tau} V\left(t_{0}\right) \leq e^{2 \lambda \tau} V\left(t_{0}\right),
$$

which implies that

$$
\|y(t)\| \leq M\left\|y\left(t_{0}\right)\right\|, \quad t \geq t_{0},
$$

where $M=e^{\lambda \tau}$

The proof of Theorem 7 is complete.

\section{Applications}

The following illustrative example will demonstrate the effectiveness of our results.

Example 8. Consider the following impulsive neural networks:

$$
\begin{aligned}
\dot{x}_{1}(t)= & -a_{1}(t) x_{1}(t)+b_{11}(t) f_{1}\left(x_{1}(t)\right) \\
& +b_{12}(t) f_{2}\left(x_{2}(t)\right)+I_{1}(t), \\
\dot{x}_{2}(t)= & -a_{2}(t) x_{2}(t)+b_{21}(t) f_{1}\left(x_{1}(t)\right) \\
& +b_{22}(t) f_{2}\left(x_{2}(t)\right)+I_{2}(t), \\
x_{1}\left(t_{k}\right)= & \left(1+\sigma_{1 k}\right) x_{1}\left(t_{k}^{-}\right)-\sigma_{1 k}, \quad k \in \mathbb{Z}_{+}, \\
x_{2}\left(t_{k}\right)= & \left(1+\sigma_{2 k}\right) x_{2}\left(t_{k}^{-}\right)-\sigma_{2 k}, \quad k \in \mathbb{Z}_{+},
\end{aligned}
$$

where $f_{1}(u)=f_{2}(u)=(|u+1|-|u-1|) / 2$.

It is easy to see that $f_{j}, j=1,2$ satisfying hypothesis $\left(\mathrm{H}_{1}\right)$ with $l_{i}=l_{2}=1$. We have $a_{1}(t)=3, a_{2}(t)=2-(3 / 2) \cos (2 t)$, $b_{11}(t)=|\sin t| / 2, b_{12}(t)=1 / 2, b_{21}(t)=2, b_{22}(t)=-1-$ $(\cos (2 t) / 2), I_{1}=(5 / 2)-(|\sin t| / 2), I_{2}=1-\cos (2 t), \sigma_{1 k}=$ $\sqrt{1+\left(1 / 5 k^{2}\right)}-1, \sigma_{2 k}=\sqrt{1+\left(1 / 6 k^{2}\right)}-1$. 
Let $\alpha=3$. It can be deduced that $\alpha I-A(t)+B(t) L \leq$ 0 and $\prod_{k=1}^{\infty} \max _{i=1,2}\left|1+\sigma_{i k}\right|<\infty$. Hence, all the conditions of Theorem 2 are satisfied; then the equilibrium point $x^{\star}=$ $(1,1)$ of the above system (31) is globally exponentially stable with the given convergence rate $\alpha=3$.

\section{Acknowledgments}

This work was jointly supported by the Project of Shandong Province Higher Educational Science and Technology Program (J12LI04), Research Fund for Excellent Young and Middle-aged Scientists of Shandong Province (BS2012DX039), and National Natural Science Foundation of China $(11226136,11171192)$.

\section{References}

[1] Z. Yang and D. Xu, "Stability analysis of delay neural networks with impulsive effects," IEEE Transactions on Circuits and Systems II, vol. 52, no. 1, pp. 517-521, 2005.

[2] J. Cao, "Global stability analysis in delayed cellular neural networks," Physical Review E, vol. 59, no. 5, pp. 5940-5944, 1999.

[3] J. Shen, Y. Liu, and J. Li, "Asymptotic behavior of solutions of nonlinear neutral differential equations with impulses," Journal of Mathematical Analysis and Applications, vol. 332, no. 1, pp. 179-189, 2007.

[4] B. Kosko, Neural Networks and Fuzzy Systems, Prentice Hall, New Delhi, India, 1992.

[5] J. Cao, "On stability of delayed cellular neural networks," Physics Letters A, vol. 261, no. 5-6, pp. 303-308, 1999.

[6] K. Gopalsamy, "Stability of artificial neural networks with impulses," Applied Mathematics and Computation, vol. 154, no. 3, pp. 783-813, 2004.

[7] R. Samidurai, S. Marshal Anthoni, and K. Balachandran, "Global exponential stability of neutral-type impulsive neural networks with discrete and distributed delays," Nonlinear Analysis: Hybrid Systems, vol. 4, no. 1, pp. 103-112, 2010.

[8] B. Lisena, "Exponential stability of Hopfield neural networks with impulses," Nonlinear Analysis: Real World Applications, vol. 12, no. 4, pp. 1923-1930, 2011.

[9] S. Wu, C. Li, X. Liao, and S. Duan, "Exponential stability of impulsive discrete systems with time delay and applications in stochastic neural networks: a Razumikhin approach," Neurocomputing, vol. 82, pp. 29-36, 2012.

[10] A. Berman and R. J. Plemmons, Nonnegative Matrices in The Mathematical Sciences, Academic Press, New York, NY, USA, 1979.

[11] D. D. Bainnov and P. S. Simeonov, Systems with Impulsive Effect Stability Theory and Applications, Halsted Press, New York, NY, USA, 1989.

[12] V. Lakshmikantham, D. D. Baŭnov, and P. S. Simeonov, Theory of Impulsive Differential Equations, World Scientific, Singapore, 1989.

[13] X. L. Fu, B. Q. Yan, and Y. S. Liu, Introduction of Impulsive Differential Systems, Science Press, Beijing, China, 2005.

[14] I. M. Stamova, Stability Analysis of Impulsive Functional Differential Equations, Walter de Gruyter, New York, NY, USA, 2009.

[15] X. Liu and Q. Wang, "The method of Lyapunov functionals and exponential stability of impulsive systems with time delay," Nonlinear Analysis: Theory, Methods and Applications, vol. 66, no. 7, pp. 1465-1484, 2007.
[16] X. D. Li, "New results on global exponential stabilization of impulsive functional differential equations with infinite delays or finite delays," Nonlinear Analysis: Real World Applications, vol. 11, no. 5, pp. 4194-4201, 2010.

[17] X. Z. Liu, "Stability of impulsive control systems with time delay," Mathematical and Computer Modelling, vol. 39, no. 4-5, pp. 511-519, 2004.

[18] X. Fu and X. Li, "Global exponential stability and global attractivity of impulsive Hopfield neural networks with time delays," Journal of Computational and Applied Mathematics, vol. 231, no. 1, pp. 187-199, 2009.

[19] X. Li, X. Fu, P. Balasubramaniam, and R. Rakkiyappan, "Existence, uniqueness and stability analysis of recurrent neural networks with time delay in the leakage term under impulsive perturbations," Nonlinear Analysis: Real World Applications, vol. 11, no. 5, pp. 4092-4108, 2010.

[20] X. D. Li and Z. Chen, "Stability properties for Hopfield neural networks with delays and impulsive perturbations," Nonlinear Analysis: Real World Applications, vol. 10, no. 5, pp. 3253-3265, 2009.

[21] X. D. Li and M. Bohner, "Exponential synchronization of chaotic neural networks with mixed delays and impulsive effects via output coupling with delay feedback," Mathematical and Computer Modelling, vol. 52, no. 5-6, pp. 643-653, 2010.

[22] H. Akça, R. Alassar, V. Covachev, Z. Covacheva, and E. AlZahrani, "Continuous-time additive Hopfield-type neural networks with impulses," Journal of Mathematical Analysis and Applications, vol. 290, no. 2, pp. 436-451, 2004.

[23] Z. T. Huang, Q. G. Yang, and X. S. Luo, "Exponential stability of impulsive neural networks with time-varying delays," Chaos, Solitons and Fractals, vol. 35, no. 4, pp. 770-780, 2008.

[24] I. M. Stamova and R. Ilarionov, "On global exponential stability for impulsive cellular neural networks with time-varying delays," Computers and Mathematics with Applications, vol. 59, no. 11, pp. 3508-3515, 2010. 

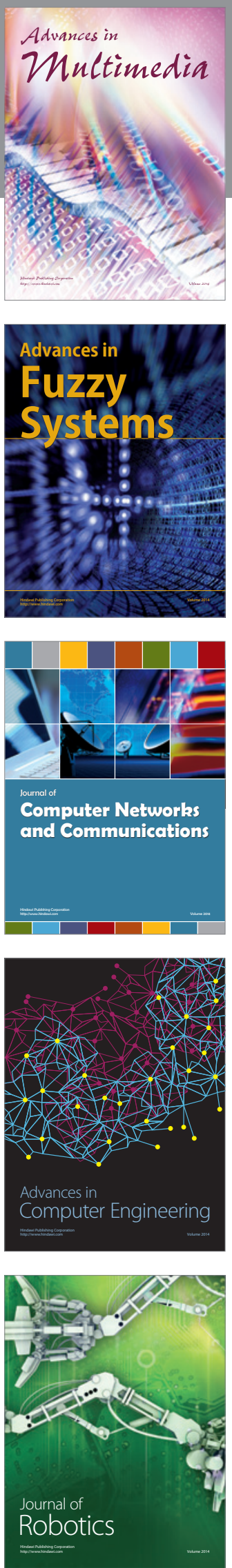

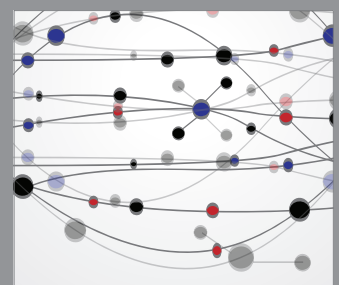

The Scientific World Journal
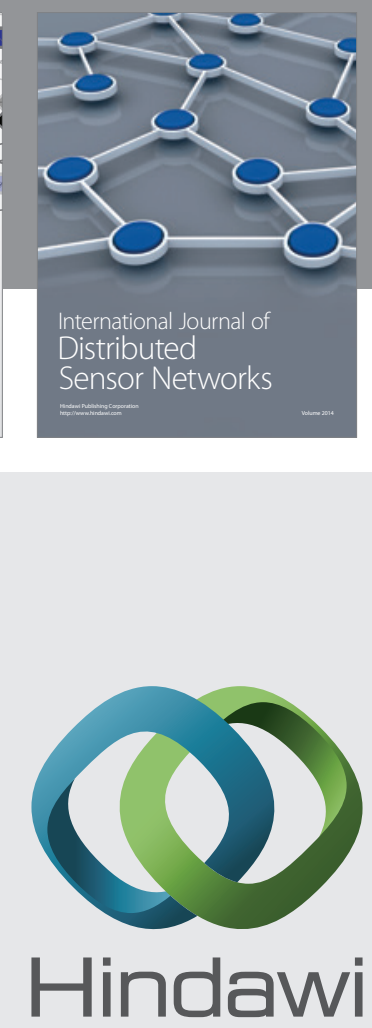

Submit your manuscripts at

http://www.hindawi.com
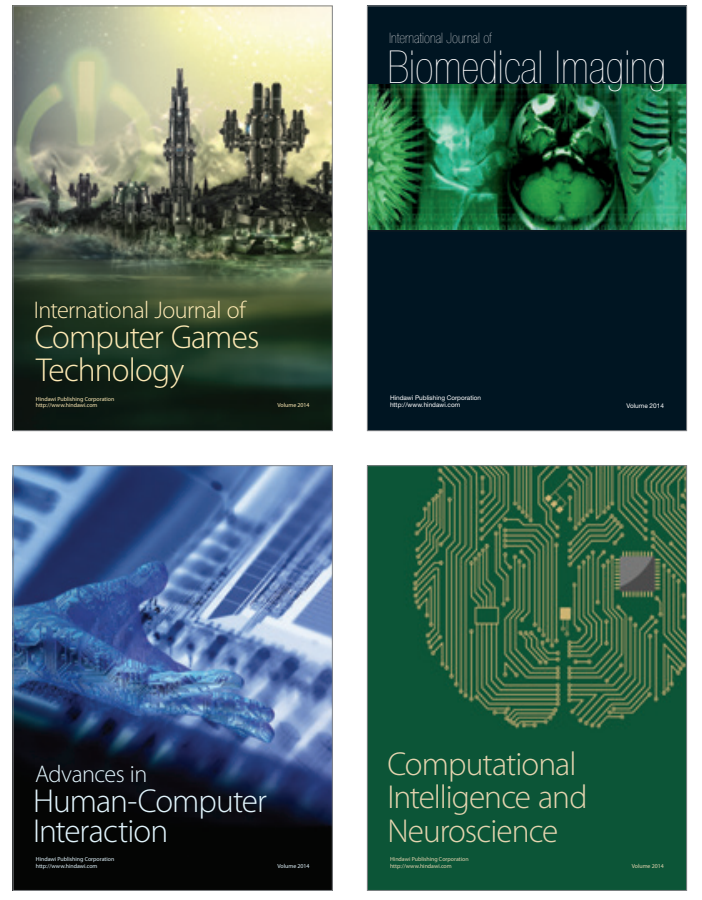
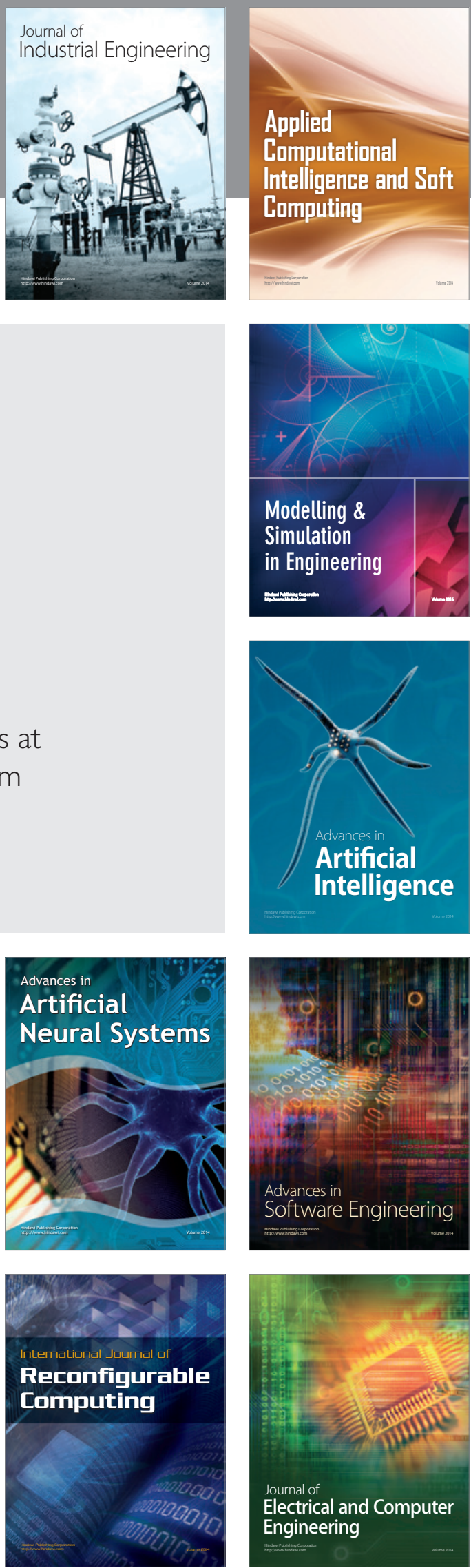